\title{
Hybrid Cooperation through Full-Duplex Opportunistic Relaying and Max-Link Relay Selection with Transmit Power Adaptation
}

\author{
Nikolaos Nomikos, Themistoklis Charalambous, Ioannis Krikidis, Demosthenes Vouyioukas and Mikael Johansson
}

\begin{abstract}
In this work, we study a cooperative network with multiple full-duplex buffer-aided relays. A hybrid cooperative relaying policy is proposed that employs power adaptation and consists of two alternative schemes: (i) full-duplex transmission through the relay which requires the least total power expenditure and loop interference is mitigated through power adaptation; (ii) buffer-aided $\max -$ link selection with power adaptation, when full-duplexity is not feasible. Aiming to reduce the overhead of channel state information (CSI) acquisition and processing, we propose a suboptimal distributed method for relay selection, for which the network performance is not degraded significantly. We show that power adaptation offers reduced overhead of CSI acquisition. Numerical results and comparisons with other state-of-the-art relaying schemes are provided and performance evaluation in terms of throughput, power minimization and switching rate, show the benefits of the proposed hybrid scheme.
\end{abstract}

Index Terms-Cooperative networks, full-duplex, opportunistic relaying, buffer-aided relays, hybrid relay selection.

\section{INTRODUCTION}

Wireless communications have been a driving factor towards the ubiquitous connectivity which is experienced by the mobile users nowadays. In order to maintain the Quality of Service (QoS) in such a level so as to achieve data rates in the order of Gbps, as targeted by the fourth generation (4G) networks and the imminent fifth generation (5G) networks, service degradation due to multipath fading, pathloss and shadowing needs to be efficiently combated. The communication paradigm of cooperative relaying has received a lot of attention due to its effectiveness in alleviating these exact effects. Various protocols have been presented in [1] where the gains in transmit and receive diversity were studied. In multirelay networks, the simultaneous transmissions by the relays require equal orthogonal channels and advanced receivers so, opportunistic relay selection has been suggested in [2] to improve the resource utilization and to reduce the hardware complexity. Stemming from the relay selection concept, many works have proposed improved selection techniques [3], [4].

N. Nomikos and D. Vouyioukas are with the Department of Information and Communication Systems Engineering, University of the Aegean, Karlovassi 83200, Samos, Greece (E-mails: \{nnomikos, dvouyiou\} @aegean.gr).

T. Charalambous and M. Johansson are with the Automatic Control Lab, Electrical Engineering Department and ACCESS Linnaeus Center, Royal Institute of Technology $(\mathrm{KTH})$, Stockholm, Sweden (Emails: \{themisc, mikaelj\} @kth.se).

I. Krikidis is with the Department of Electrical and Computer Engineering, University of Cyprus, Nicosia 1678 (E-mail: krikidis@ucy.ac.cy).
Aiming to improve the performance of opportunistic relay selection, the addition of buffers at the relays unleashed novel selection policies. Some of them are discussed in the sequel. In [5] max - max relay selection has been proposed where the relay that receives the source signal is not necessarily the one that forwards information to the destination, as another relay with packets in its buffer may experience more favorable conditions. Furthermore, in [6] increased degrees of freedom are offered to the selection process as the link with the best quality is activated among the $2 K$ ones, in a network with $K$ relays, each one providing a source-relay $(S R)$ and a relaydestination $(R D)$ link. In addition, regardless of buffering capabilities, all the above schemes consider half-duplex (HD) relays where reception and transmission occur separately, resulting in resource loss in time and/or frequency. In order to alleviate this loss, buffer-aided successive schemes have been proposed where two relays are concurrently activated, one for reception and the other for transmission, thus mimicking full-duplexity but at the cost of inter-relay interference (IRI). Works from this field include [7] where inter-relay interference was considered negligible and [8], [9] where IRI was mitigated to some extent through avoidance or cancellation. These studies have shown improved performance and the half-duplex loss has been recovered to a significant extend.

In a different line of research, full-duplex (FD) relays have been employed, in order to increase the spectral efficiency of relay networks. As HD relays require twice the resources in time (or frequency) to achieve the same spectral efficiency as FD relays, a lot of articles propose techniques where FD relaying outperforms HD relaying. Most of these articles focus on loop-interference (LI) mitigation techniques which is the main detrimental factor in FD relay networks as the relay is transmitting and receiving simultaneously, thus introducing LI from its output antenna to its input antenna. The work in [10] illustrates antenna isolation as well as interference cancellation and suppression techniques which result in LI levels which end up similar to noise levels. The investigation of hardware constraints at the receiver and imperfect CSI in FD multiantenna networks have been the subjects of [11]. Numerical evaluation of the effects of the limited dynamic range and the residual LI are provided as well as a rate-maximization transmission scheme where a power constraint is imposed.

The two aforementioned lines of research cross at the 
adoption of a hybrid combination of FD and HD relaying where switching to either mode is performed according to specific performance criteria. In [12] hybrid FD-HD relaying with power adaptation has been presented where instantaneous and statistical CSI are considered when selecting the appropriate duplexing mode for a single relay network. Also, the availability of the source-destination $(S D)$ link, as well as both Amplify-and-Forward (AF) and Decode-and-Forward (DF) protocols are considered. The proposed hybrid scheme chooses among FD, HD or direct transmission based on the achieved capacity of each mode. Another work [13] examined opportunistic FD AF relay selection, including a hybrid FD/HD relay scheme which aims at capacity maximization. While these works achieved improved performance via the combination of robust HD relaying with spectral efficient FD relaying, no buffering was considered at the relays. As a result, their HD scheme lacked the characteristics of increased diversity that buffer-aided relays offer and the overall performance was constrained; more importantly, they targeted capacity maximization without considering the power expenditure by the source and the relays.

In this work, a hybrid relay selection scheme is proposed, herein called the HMP, which employs FD buffer-aided relays, for the first time in a relay selection scheme. The proposed selection policy takes the decision to activate a relay that satisfies a QoS requirement, herein expressed by a threshold - called the capture ratio - in Signal-to-Noise Ratio (SNR) for successful decoding, while at the same time the power expenditure is minimized. First, this is done jointly for the $S R$ and $R D$ links. If none of the relays can establish the QoS requirements, a single-link is selected that satisfies the requirements. Since the process of hybrid selection is based on a costly instantaneous CSI acquisition, a suboptimal version is proposed, herein called the sHMP, in which the previously selected relay is first examined on whether it satisfies the QoS requirement within a power expenditure margin; if it does, the source-relay-destination $(S R D)$ communication continues through this relay; otherwise, the CSI acquisition process is triggered again. The contribution of this work is threefold:

(i) FD buffer-aided relaying has been considered for the first time in a relay selection scheme. This has been combined with HD buffer-aided relaying in order to form hybrid relaying schemes where the improved spectral resource usage of FD relaying is successfully amalgamated with LI-free, robust HD buffer-aided relaying. In order to achieve improved network performance, we prioritize the FD mode of operation targeting a specific capture ratio. However, whenever this is not possible due to increased LI, or, if both $S R$ and $R D$ cannot be above the required SNR value, robust single-link selection, based on the max - link selection policy of [6] is employed.

(ii) Power adaptation has been used in our relaying scheme in order to choose the relay which satisfies the QoS requirement at the minimum power expenditure while at the same time it allows LI mitigation as the transmitted

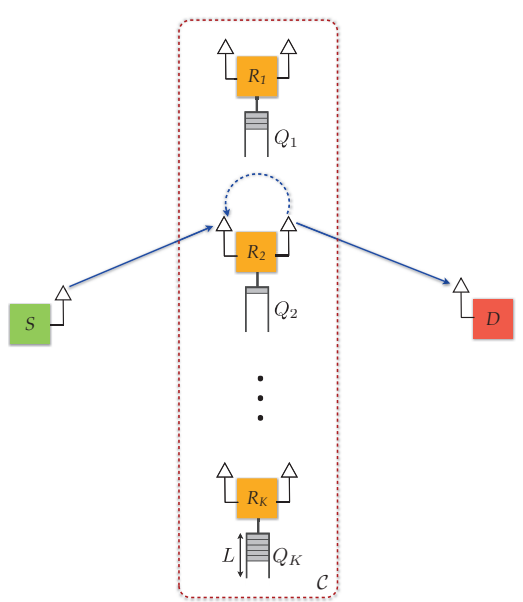

Fig. 1. A cooperative network consisting of a source $S, K$ full-duplex relays which form a clustered topology (denoted by $\mathcal{C}$ ) and a destination $D$.

power of the relay is set at the lowest possible level for the target capture ratio, thus reducing the amount of interference at the receiving antenna of the relay.

(iii) Finally, we propose a suboptimal distributed method for the hybrid relay selection that does not deteriorate the network performance significantly, but reduces the overhead of CSI acquisition and processing tremendously, since it avoids constant switching among the relays. It turns out that power adaptation improves the switching rate, reducing the instances CSI acquisition is necessitated.

The remainder of this paper is as follows. In Section II, the system model is outlined, while Section III defines the basic elements of our study. Section IV describes in detail the proposed hybrid relaying scheme as well as its suboptimal version and gives insight on the switching rate reduction which is achieved. The performance of the proposed algorithm in terms of outage, average throughput and delay, along with comparisons with other state-of-the-art relaying schemes are presented in Section V. Finally, conclusions and a discussion on future directions are included in Section VI.

\section{SYSTEM MODEL}

In the context of this study, a cooperative network consisting of one source $S, K$ DF relays which form a clustered topology denoted by $\mathcal{C}$ and one destination $D$ is assumed, as shown in Fig. 1. Each relay $R_{k} \in \mathcal{C}$ is equipped with two antennas, one for reception and one for transmission and are able to operate in FD mode where simultaneous reception and transmission is feasible. To simplify the analysis, we examine the case where connectivity between the source and the destination is established only via the relays and so, the $S D$ link is ignored.

Moreover, the relays have buffers to support the hybrid operation of the proposed scheme. Each buffer $Q_{k}$ has a capacity of $L$ data elements which are measured in bits-perchannel-use (BPCU) and the parameter $l_{k} \in \mathbb{Z}_{+}, l_{k} \in[0, L]$ denotes the number of data elements in $Q_{k}$. Assuming a fixed transmission rate $r_{0}$ BPCU by the source and the relay $R_{k}$, $l_{k}$ is increased by $r_{0}$ BPCU when the source transmits to $R_{k}$, and when the relay $R_{k}$ transmits towards the destination, $l_{k}$ is 
reduced by $r_{0}$. At the beginning of the network's operation, the buffers of the relays are empty i.e. $l_{k}=0$ for all $\mathrm{k}$.

The operation is assumed to be divided into time-slots, where in each slot only a relay supports the communication either through FD relaying or max - link selection. Regardless of the adopted strategy, the source's transmission power is denoted by $P_{S}$ and the relay's by $P_{R_{k}}$. The source $S$ is assumed to be saturated and hence, it has always data to transmit. An acknowledgment/negative-acknowledgment (ACK/NACK) mechanism is employed, where short-length error-free packets are sent by the receivers over a separate narrow-band channel.

All wireless links exhibit fading and we assume an Additive White Gaussian Noise (AWGN) channel. The fading is assumed to be stationary, with frequency non-selective Rayleigh block fading. This means that the fading coefficients $h_{i j}$ (for the $i \rightarrow j$ link) remain constant during one slot, but change independently from one slot to another according to a circularly symmetric complex Gaussian distribution with zero mean and unit variance. The channel gains are $g_{i j}=\left|h_{i j}\right|^{2}$ and exponentially distributed. Also, the circular symmetric complex Gaussian noise $N$ has zero mean and variance $n$ (i.e., $N \sim \mathcal{C N}(0, n))$ and, for simplicity, is assumed to be equal at each receiver.

Here, global CSI is considered to be available but the proposed scheme can be straightforwardly implemented with various centralized and distributed CSI and relay selection approaches as in [2] where each relay sets a timer in accordance to the channel quality and through a countdown process the best relay is selected. Additionally, CSI overhead can be reduced significantly, through distributed-switch-and-staycombining (DSSC) as in [14], [15]. In these works, distributed relay and relay-pair selection were presented and in each transmission phase, if a targeted spectral efficiency was still achieved by the previously selected relay(s), then a new round of CSI acquisition and processing was avoided.

As FD operation is supported by the relays, LI degrades the network's performance. To account for this fact, $h_{R_{k} R_{k}}$ denotes the instantaneous residual LI between the two antennas of relay $R_{k}$, which follows a complex Gaussian distribution and takes values in the range $\left(0, \sigma_{R_{k} R_{k}}^{2}\right)$.

\section{Preliminaries}

Due to battery limitations, we assume that each transmitting node $i$ (source and relays) has a maximum peak power $P_{i}^{\max }$. The power constraint is, in general, a critical design parameter in relay networks [16].

Assuming a Gaussian input distribution and an information theoretic capacity achieving channel coding scheme, the instantaneous Signal-to-Interference-and-Noise Ratio (SINR) from $S$ to $R_{k}$ and the instantaneous SNR from $R_{k}$ to $D$ when relay $R_{k}$ is selected, are expressed as

$$
\frac{g_{S R_{k}} P_{S}}{g_{R_{k} R_{k}} P_{R_{k}}+n} \geq \gamma_{0}
$$

and

$$
\frac{g_{R_{k} D} P_{R_{k}}}{n} \geq \gamma_{0}
$$

respectively. When inequalities (1) and (2) are not satisfied simultaneously by any of the $K$ relays, the proposed scheme switches to the single-link selection scheme, namely the max - link that incorporates power adaptation. In the singlelink mode, there is no LI between relays and hence, the SINR expression in (1) becomes

$$
\frac{g_{S R_{k}} P_{S}}{n} \geq \gamma_{0} .
$$

On the other hand, if $R_{k}$ is selected to forward a packet from its buffer towards the destination, the SNR remains the same as in equation (2).

\section{HYBRID MINIMUM POWER (HMP) RELAY SELECTION}

In this Section, we present the proposed hybrid relay selection scheme which is based on switching among FD relay selection and max - link (ML) selection. We name the optimal version as HMP and its suboptimal version as sHMP. Also, power adaptive transmissions are performed in order to select the relay which will establish the $S R D$ communication with the minimum power cost.

\section{A. Optimal full-duplex relaying with max - link selection}

In an arbitrary time-slot $t$, the possibility of employing FD relaying is first investigated by having the $K$ relays acquiring CSI and calculating the total power required by both the source and each relay, in order to satisfy the QoS requirements (2) and (1) at the destination and the relay, respectively. Denote by $\mathcal{F}$ the set of relays that can employ FD operation, by satisfying QoS requirements (2) and (1). In the FD mode, LI arises between the relay antennas. As a result, the relay first calculates the minimum power $P_{R_{k}}^{*}$ via (2) required for successful transmission to $D$, i.e.,

$$
P_{R_{k}}^{*}=\frac{\gamma_{0} n}{g_{R_{k} D}} .
$$

If, $P_{R_{k}}^{*} \geq P_{R_{k}}^{\max }$, FD operation for relay $k$ cannot be supported and there is no need to compute the power needed by the source. Otherwise, if $P_{R_{k}}^{*} \leq P_{R_{k}}^{\max }, R_{k}$ provides this information to the source together with the CSI of the $h_{R_{k} R_{k}}$, so that the source can compute $P_{S_{k}}{ }^{1}$. The minimum power $P_{S_{k}}^{*}$ needed for successful decoding at the specific relay is then found via (1), i.e.,

$$
P_{S_{k}}^{*}=\frac{\gamma_{0}\left(g_{R_{k} R_{k}} P_{R_{k}}^{*}+n\right)}{g_{S R_{k}}} .
$$

If, in addition, $P_{S_{k}}^{*} \leq P_{S}^{\max }$, then FD operation is supported and relay $k$ is added to set $\mathcal{F}$. Next, the source selects the FD relay, denoted $R_{b}^{(F D)}$, that requires the minimum total power to satisfy the QoS requirements, set via the capture ratio $\gamma_{0}$. The selection process is written as

$$
b^{(F D)}=\arg \min _{k \in \mathcal{F}}\left(P_{S_{k}}^{*}+P_{R_{k}}^{*}\right) .
$$

If set $\mathcal{F}=\emptyset$ (i.e., none of the relays fulfills (2) and (1) within the power limits), then HMP switches to the

\footnotetext{
${ }^{1}$ Note that the source power may differ for each relay. For this reason, we have $P_{S_{k}}$ to denote the power when relay $k$ is considered.
} 
max - link relay selection policy. Denote by $\mathcal{M}$ the set of links that can be employed by the max - link relay selection policy; this set consists of the following two subsets: subset $\mathcal{T} \subseteq \mathcal{M}$, which includes all the relays for which their buffer is not empty and hence able to transmit to the destination, and subset $\mathcal{A} \subseteq \mathcal{M}$, which includes all the relays for which their buffer is not full and they are available to receive a packet from the source. Note that $\mathcal{A} \cup \mathcal{T}=\mathcal{M}$.

The additional characteristic here is again power adaptation, where the minimum powers $P_{S_{k}}^{\dagger}$ and $P_{R_{k}}^{\dagger}$ are computed via (3) and (2) for the $S R$ links for $R_{k} \in \mathcal{A}$ and for the $R D$ links for $R_{k} \in \mathcal{T}$, respectively. These minimum powers are compared with $P_{S_{k}}^{\max }$ and $P_{R_{k}}^{\max }$, respectively; the links that require power which is less than or equal to the maximum available power of the source or the relay, can be potentially activated and hence, are included in set $\mathcal{M}$. Thus, the selection of $R_{b}^{(M L)}$ is written as

$$
b^{(M L)}=\arg \min _{k \in \mathcal{M}} \min \left(P_{S_{k}}^{\dagger}, P_{R_{k}}^{\dagger}\right),
$$

as the selected relay is the one that requires the minimum power among the $S R$ and $R D$ links in $\mathcal{M}$.

Remark 1. Note that, while the single-link relaying scheme is similar to max - link, our scheme includes power adaptation enabling the minimization of the power used for transmission. More specifically, the power adaptation which is performed in accordance to the SINR requirements at each link, is equivalent to the minimization of the total power in the network subject to the SINR constraints (see, for example, [17], [18]). As a result, the selection of the best pair minimizes the energy spent to satisfy the SINR constraint. As a result, in each time-slot the least amount of power is required in order to achieve the SNR target $\gamma_{0}$.

\section{B. Suboptimal full-duplex relaying with max - link selection}

From the previous description, it is obvious that the continuous calculation of the required powers for each $S R D$ branch increases the CSI overhead significantly. So, we propose sHMP, where DSSC is adopted in the selection process. Through the use of DSSC, the same relay can establish the $S R D$ communication for more time-slots as long as it satisfies a threshold which is linked to a specific metric, such as the achieved SNR or the total power expenditure. As a result, sHMP reduces the instances of CSI acquisition and processing which are required for relay selection. As power minimization is the main target of the proposed selection scheme, we impose a maximum power expenditure threshold for sHMP. We denote this threshold by $Z_{j}$ and it can be associated with the total power limits of the devices (source and relay), or, it could be a parameter that aims to monitor the lifetime of the network and as a result, this parameter could be made known to all the nodes at the beginning of the network's operation; for simplicity of exposition, we let $Z_{j}$ to be the same for all nodes, i.e., $Z_{j}=Z, \forall j$.

At the beginning of its operation, the selection of the best relay is performed as described for the optimal hybrid relay selection policy. Suppose that in an arbitrary time-slot $t$ the selection of the best relay is performed as in HMP. The differentiation in the suboptimal version of the algorithm emerges from the fact that at time-slot $t+1$, the previous best relay (selected to operate in full-duplex mode) is examined first, checking whether it can establish the $S R D$ connection using a total power expenditure which is less or equal to a threshold, $Z$. If the relay fulfills the condition, then it is employed again for transmission. As a result, the selection process in the $t+1$ time-slot is expressed as

$$
b^{(t+1)}=\left\{\begin{array}{l}
R_{j} \mid R_{j} \in \mathcal{F}, P_{S_{j}}^{*}+P_{R_{j}}^{*} \leq Z, \\
b^{(F D)}, \text { otherwise. }
\end{array}\right.
$$

where $R_{j}$ is the relay with index $j$ which was selected to assist in FD mode in time-slot $t$ and $b^{(F D)}$ denotes the selection process as expressed by (6). Note that in the case, for which at a slot the network operates in a single-link mode, CSI acquisition is triggered in the next slot.

The selection process described by (8) assures that CSI acquisition is avoided as long as full-duplexity is achieved at a power cost which is not higher than $Z$. This scheme trades-off complexity reduction for performance degradation in terms of power reduction and allows the network to be configured in a variety of ways. For example, $Z$ can be adjusted in such a way as to avoid CSI acquisition by setting a higher value for $Z$ when battery-dependent relays have enough energy, or by imposing a more strict $Z$ when the total power expenditure needs to be minimized as much as possible ${ }^{2}$.

Remark 2. Since the work in [12] aims at maximizing the capacity, the source power is fixed (and equal to $P_{S}^{\max }$ ) and only the relay power is adjusted (in this simple yet illustrative three-node network). However, in our work, we aim to minimize the power expenditure, and hence both power levels are adjusted so that the total power is minimized. The benefits of power adaptation in such a scenario are well presented in [12].

If we consider algorithms that do not use power adaptation (i.e., they just fix their transmission power), or even the approach in [12] (where the source uses maximum power and only the power of the relay is adjusted), then it would be difficult to share efficiently $Z$ between the source and the selected relay. Hence, this would result to more switchings forcing the network for more instances of CSI acquisition.

Based on the condition given in Eq. (8), $Z$ can be split into two power levels, namely $Z_{1}$ and $Z_{2}$ (i.e., $Z_{1}+Z_{2}=Z$ ): one for the $S R_{k}$ link and one for the $R_{k} D$ link. For the $R_{k} D$ link, since there is no interference, the condition is fulfilled if $P_{R_{j}}^{*} \leq Z_{2}$. As a result, by Eq. (4) it is required that

$$
g_{R_{k} D} \geq \frac{\gamma_{0} n}{Z_{2}} .
$$

Similarly, for the $S R_{k}$ link the corresponding condition is

\footnotetext{
${ }^{2}$ We note however, that the power cost of CSI acquisition is not considered in the analysis and the results.
} 
fulfilled if $P_{S_{j}}^{*} \leq Z_{1}$. Since, power adaptation is adopted, in the case $P_{R_{j}}^{*} \leq Z_{2}$, we substitute $P_{R_{j}}^{*}$ found in Eq. (4) to Eq. (5) and thus,

$$
g_{S R_{k}} \geq \frac{\gamma_{0}\left(\frac{g_{R_{k} R_{k}}}{g_{R_{k} D}} \gamma_{0}+1\right) n}{Z_{1}} .
$$

However, if there is no power adaptation, the powers should be chosen a priori, say $\left(P_{S_{j}}^{*}, P_{R_{j}}^{*}\right)=\left(P_{1}, P_{2}\right)$ for all time instants; then Eqs. (4) and (5) become

$$
\begin{aligned}
g_{R_{k} D} & \geq \frac{\gamma_{0} n}{P_{2}}, \\
g_{S R_{k}} & \geq \frac{\gamma_{0}\left(g_{R_{k} R_{k}} P_{2}+n\right)}{P_{1}} .
\end{aligned}
$$

For sustaining the same $S R D$ link, we require that both conditions (11a)-(11b) are satisfied. Hence $\left(P_{1}, P_{2}\right)$ are chosen such that

$$
\begin{gathered}
\max _{P_{1}, P_{2}} \min (\mathbb{P}((11 \mathrm{a})), \mathbb{P}((11 \mathrm{~b}))), \\
\text { s.t } P_{1}+P_{2} \leq Z, \\
P_{1}, P_{2}>0 .
\end{gathered}
$$

$\mathbb{P}((11 \mathrm{a}))$ can be expressed easily, since $g_{R_{k} D}$ is exponentially distributed (i.e., $g_{R_{k} D} \sim \operatorname{Exp}(\nu), \nu>0$ ). In addition, (11b) can be written as $g_{S R_{k}} P_{1}-g_{R_{k} R_{k}} \gamma_{0} P_{2} \geq \gamma_{0} n$. This linear combination of exponentially distributed variates can be shown (omitted due to space limitations) to have the following distribution:

$$
f_{X}(x)=\frac{\lambda \mu}{\gamma_{0} P_{2} \lambda+P_{1} \mu} \begin{cases}\exp \left(-\frac{\mu}{\gamma_{0} P_{2}} x\right), & \text { if } x \geq 0, \\ \exp \left(\frac{\lambda}{P_{1}} x\right), & \text { if } x<0 .\end{cases}
$$

where $g_{S R_{k}} \sim \operatorname{Exp}(\lambda), g_{R_{k} R_{k}} \sim \operatorname{Exp}(\mu), \lambda, \mu>0$ and $X=$ $g_{S R_{k}} P_{1}-g_{R_{k} R_{k}} \gamma_{0} P_{2}$. Hence, having statistical knowledge about the channel states, allows for computing the optimal power levels, for the case the power cannot be adapted. Note, however, that any algorithm that adopts the optimal power levels performs worse than our case, where power is adapted. Nevertheless, this optimization can be used in scenarios where only statistical information about the channels in known.

\section{Numerical Results}

In order to evaluate the performance of the HMP and sHMP schemes, we have developed a simulation setup in MATLAB $^{\circledR}$ based on the system model description in Section II. A fixed transmission rate of $2 \mathrm{BPCU}$ is assumed and the average throughput, the achievable power reduction and the relay switching rate are evaluated. Moreover, we compare the proposed scheme with other state-of-the-art relay selection schemes which include a standalone full-duplex scheme with power adaptation capabilities (FD) and various HD schemes such as best-relay selection (BRS) [2], hybrid relay selection which combines max - max and BRS [5] and max - link selection [6]. In the comparisons, we assume a network with $K=3$ relays, $r_{0}=2 \mathrm{BPCU}$ and relay buffer size $L=100$. The powers of the $S R, R D$ and $R R$ links are assumed equal i.e. we have $g_{S R}=g_{R R}=g_{R D}$.

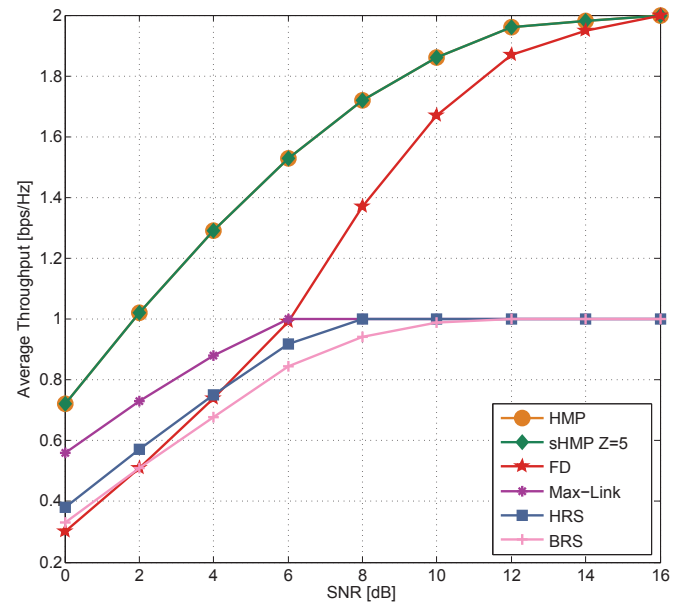

Fig. 2. Average throughput for increasing transmit SNR and different relay selection policies.

First, we examine the average throughput and the performance of each scheme is depicted in Fig.2. It is obvious that the FD schemes can achieve twice the throughput of the HD schemes. More specifically, as the available transmit power increases, power adaptation allows the LI to be efficiently mitigated and after $14 \mathrm{~dB}$ the target of $2 \mathrm{BPCU}$ is reached. In addition, HMP offers better results in the whole SNR range as it is more robust than standalone FD which lacks diversity for low SNR values and experiences outages. Also, the two versions of the proposed scheme perform identically as sHMP reverts to HMP when $Z$ is not satisfied. On the other hand, the group of HD schemes achieve a throughput of $1 \mathrm{BPCU}$ as each transmission splits the time-slots into two orthogonal sub-slots where $S R$ and $R D$ communication occurs. Finally, max - link achieves better results as it avoids outages due to adaptive link selection, in contrast to HRS and BRS.

Next, results for the power reduction performance of HMP and sHMP are illustrated in Fig. 3 for various power expenditure thresholds. The optimal version is the upper-bound as selection aims for the relay which spends in each time-slot, the least amount of power. We see that the performance of sHMP depends on the value of $Z$ and as it increases, there is a gap with the upper-bound. We remark that a more relaxed $Z$ threshold allows the same relay to establish the $S R D$ connection more frequently although another relay could offer better results in the power reduction domain.

Fig. 4 shows the behavior of HMP and sHMP with respect to the switching rate. Here, sHMP can reduce significantly the occurrence of relay selection and consequently, CSI acquisition. Since we assume a fixed transmission rate of $2 \mathrm{BPCU}$, the percentage of switching among the relays is quite low for the case of $Z=20$, as usually one relay with enough transmission power can reach the spectral efficiency target without exceeding the power threshold $Z$. As $Z$ becomes more strict, the switching rate increases and comes closer to the rate which is exhibited by HMP. However, we observe that for low SNR, sHMP curves exhibit the same behavior as in this region, max - link selection is the dominant mode and relay switching is more frequent, although still lower than HMP. 


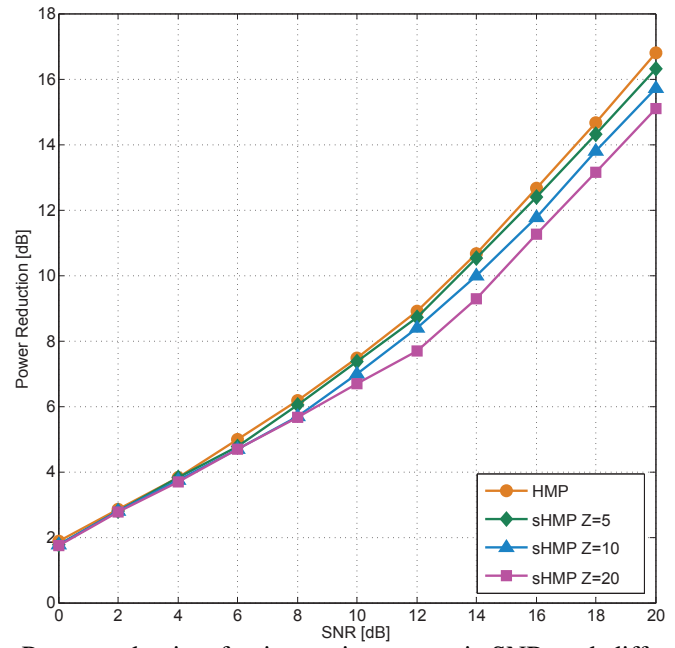

Fig. 3. Power reduction for increasing transmit SNR and different power threshold values.

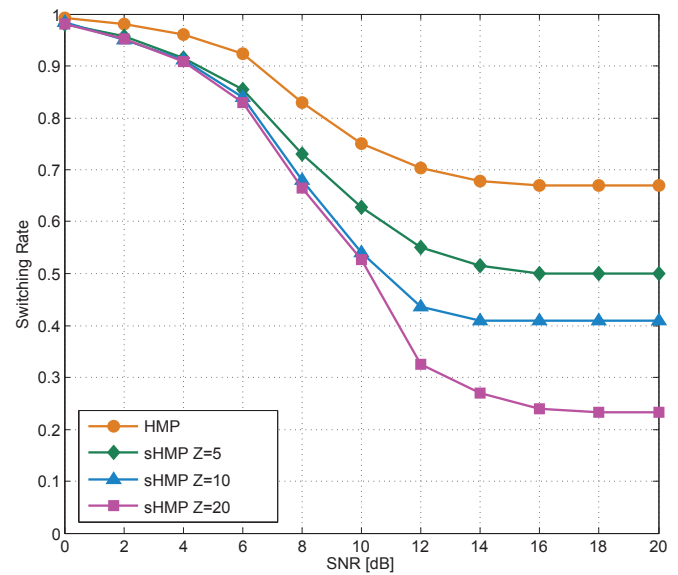

Fig. 4. Switching rates for increasing transmit SNR and different power threshold values.

\section{COnClusions And Future Directions}

In this work, we present a hybrid relay selection policy, herein called the HMP, that employs a cluster of FD bufferaided relays that can operate as HD relays in order to increase the network's robustness. FD buffer-aided relays for relay selection are being used in this work for the first time. The main characteristic of HMP is power minimization, as the selected relay achieves the QoS requirement at the minimum power cost. Furthermore, a suboptimal scheme is developed, heretofore called the sHMP, which reduces CSI overhead by imposing a power expenditure margin, which if satisfied allows the $S R D$ communication without a new relay selection round. Power adaptation in the suboptimal scheme has a considerable advantage over constant power approaches, due to the flexibility in offering more power to the worst link, thus avoiding further switching and CSI acquisition. The benefits of this network deployment are demonstrated via a numerical evaluation, where improved performance is observed regarding the average throughput, the power reduction and the switching rate.

A scheme that combines the reception of a packet on one relay and the transmission of a packet from an other relay is part of ongoing research. Finally, the effect of DSSC on delay reduction, in a combined full-duplex and buffer-aided half-duplex relaying scheme, is another interesting research direction.

\section{ACKNOWLEDGMENT}

This work was partially supported by the Research Promotion Foundation, Cyprus under the project KOYLTOYRA/BPNE/0613/04 "Full-Duplex Radio: Modeling, Analysis and Design (FD-RD)" and the Swedish Foundation for Strategic Research (SSF).

\section{REFERENCES}

[1] J. N. Laneman, D. N. C. Tse, and G. W. Wornell, "Cooperative diversity in wireless networks: Efficient protocols and outage behavior", IEEE Trans. Inform. Theory, vol. 50, pp. 3062-3080, Dec. 2004.

[2] A. Bletsas, A. Khisti, D. Reed, and A. Lippman, "A simple cooperative diversity method based on network path selection," IEEE J. Select. Areas Commun., vol. 24, pp. 659-672, March 2006.

[3] D. S. Michalopoulos and G. K. Karagiannidis, "Performance analysis of single relay selection in Rayleigh fading," IEEE Trans. Wireless Commun., vol. 7, pp. 3718-3724, Oct. 2008.

[4] I. Krikidis, J. S. Thompson, S. McLaughlin, and N. Goertz, "Max-min relay selection for legacy amplify-and-forward systems with interference," IEEE Trans. Wireless Commun., vol. 8, pp. 3016-3027, June 2009.

[5] A. Ikhlef, D. S. Michalopoulos, and R. Schober, "Max-max relay selection for relays with buffers," IEEE Trans. Wireless Commun., vol. 11, no. 3, pp. 1124-1135, March 2012.

[6] I. Krikidis, T. Charalambous, and J. S. Thompson, "Buffer-aided relay selection for cooperative diversity systems without delay constraints," IEEE Trans. Wireless Commun., vol.11, no.5, pp.1957-1967, May 2012.

[7] A. Ikhlef, K. Junsu, and R. Schober, "Mimicking full-duplex relaying using half-duplex relays with buffers," IEEE Trans. Vehicular Tech., vol. 61, no. 7, pp. 3025-3037, Sept. 2012.

[8] N. Nomikos, D. Vouyioukas, T. Charalambous, I. Krikidis, P. Makris, D. N. Skoutas, M. Johansson and C. Skianis, "Joint relay-pair selection for buffer-aided successive opportunistic relaying," Wiley-Blackwell Trans. Emerg. Telecom. Techn., 2013 (accepted).

[9] N. Nomikos, T. Charalambous, I. Krikidis, D. N. Skoutas, D. Vouyioukas, and M. Johansson, "Buffer-aided successive opportunistic relaying with inter-relay interference cancellation," IEEE Pers. Indoor. Mob. Rad. Comm. (PIMRC), London, United Kingdom, Sept. 2013.

[10] T. Riihonen, S. Werner and R. Wichman, "Mitigation of loopback selfinterference in full-duplex MIMO relays," IEEE Trans. Sign. Proc., vol. 59, no. 12, pp. 5983-5993, Dec. 2011.

[11] B. P. Day, A. R. Margetts, D. W. Bliss and P. Schniter, "Full-duplex MIMO relaying: Achievable rates under limited dynamic range," IEEE J. Select. Areas Commun., vol. 30, no. 8, pp. 1541-1553, Sept. 2012.

[12] T. Riihonen, S. Werner and R. Wichman, "Hybrid full-duplex/halfduplex relaying with transmit power adaptation," IEEE Trans. Wireless Commun., vol. 10, no. 9, pp.3074-3085, Sept. 2011.

[13] I. Krikidis, H. A. Suraweera, P. J. Smith and Chau Yuen, "Full-duplex relay selection for amplify-and-forward cooperative networks," IEEE Trans. Wireless Commun., vol. 11, no.12, pp. 4381-4393, Dec. 2012.

[14] D. S. Michalopoulos and G. K. Karagiannidis, "Selective cooperative relaying over time-varying channels," IEEE Trans. Commun., vol. 58, no. 8 pp. 2402-2412, Aug. 2010.

[15] N. Nomikos, P. Makris, D. Vouyioukas, D. N. Skoutas and C. Skianis, "Distributed joint relay-pair selection for buffer-aided successive opportunistic relaying," to be presented at IEEE Int. Works. on Comp. Aid. Mod. Anal. and Des. (CAMAD), Berlin, Germany, Sept. 2013.

[16] G. Kramer, M. Gastpar, P. Gupta, "Cooperative strategies and capacity theorems for relay networks," IEEE Trans. on Information Theory, vol. 51, no. 9, pp. 3037-3063, Sept. 2005.

[17] G. J. Foschini and Z. Miljanic, "A simple distributed autonomous power control algorithm and its convergence," IEEE Trans. Vehicular Tech., vol. 42, no. 4, Nov. 1993.

[18] T. Charalambous, "Power control in wireless ad-hoc networks: stability and convergence under uncertainties," Optimization, Simulations and Control, Springer Series in Optimization and Its Application (SOIA), vol. 76, pp. 143-174, 2013. 